(c) 2013 IEEE. Personal use of this material is permitted. Permission from IEEE must be obtained for all other uses, in any current or future media, including reprinting/republishing this material for advertising or promotional purposes, creating new collective works, for resale or redistribution

to servers or lists, or reuse of any copyrighted component of this work in other works.

\title{
Optimal technicians' allocation problem with respect failure reparation
}

\author{
Beatriz de la Cruz Miranda, Carmen Mas Machuca, Senior Member IEEE \\ Technische Universität München, Arcisstr. 21, Munich, Germany \\ e-mail: beatrizcruz88@gmail.com,cmas@tu.de
}

\begin{abstract}
The reparation of failures should be done as fast as possible to minimize the impact to the users as well as any penalties agreed in the Service Level Agreement. However, in order to minimize costs, the number of technicians as well as their locations should be minimized to keep the Total Cost of Ownership low. This compromise between cost and time has been studied within an optimization problem and different case studies have been considered
\end{abstract}

Keywords: telecommunication networks, fail, repair, fault management, reduces expenditures, optimization.

\section{INTRODUCTION}

Since the birth of the telecommunications with the telegraph appearance in 1829 to the present day, communications have undergone a major change. Alike, in this period of time society has also changed, demanding that always rising the standards of living.

The managers of telecommunication networks have been always trying to deal with the society ever increasing demand for high standards of services. However, they also try to keep their Total Benefit of Ownership (TBO), which is defined as the difference between profits and cost. Profits depend mainly on the income received from the services. These profits are limited due to the strong market competition in today's converging telecommunications market as well as to the impact of regulation. Hence to maximize TBO the best approach is to minimize costs. The cost of a telecommunication network is known as Total Cost of network Ownership (TCO). TCO has mainly two components: the capital expenditures (known as CapEx) which includes the purchase and installation of equipment and any required infrastructure; and the operational expenditures (referred as OpEx) which include any operational cost such as the maintenance of the network components, the reparation of failures, the energy costs, etc. It has been shown [1] that the cost associated to failure reparation is very important in networks and therefore, the minimization of its cost is of interest for the operators.

The goal of this paper is to evaluate quantitative and qualitatively the overall cost and time of repairing failures in a communication network. The reparation of failures should be done as fast as possible to minimize the impact to the users as well as reduce any penalties agreed in the SLA (Service Level Agreement). However, in order to minimize costs, the number of technicians as well as their locations should be minimized to keep the salary and location costs low. This problem is turned into optimization problem, looking for a commitment between time and cost. At the end, the total reparation cost and the number of technicians are presented.

This article is structured as follows. Section 2 presents the problem formulation and its implementation. Section 3 shows the application of the problem in the Germany50 network and the obtained results. Finally, Section 4 concludes the paper.

\section{PROBLEM FORMULATION}

The network problem of allocating technicians is very complex. The reparation of failures should be done as fast as possible to minimize the impact to the users. However, in order to minimize costs, the number of technicians should be minimized.

Given a network as a set of interconnected nodes with links, and given the failure rate of each node and link of the networks, the problem targets to place technician(s) at some (all) network nodes so that the failure reparation cost is minimized. The failure reparation cost is the sum of any salaries of the technicians, any cost of the location of these technicians at their assigned node, and any penalty that should be paid due to the service interruption until the failure is repaired. 
The information required to solve this problem is: the travelling time from the location of the closest technician to the failure, the distance between nodes, the lengths of the links, the time required to repair a failure in node or link, the penalty for connection interruption time, the Mean Time Between Failures (MTBF) or Failures in Time (FIT) of any node and link. The failure rate of a link depends on its length (i.e. the longer the link is, the more failures are expected to have).

It is important to stand out that the link failures are associated to the nearest node. Therefore, when a link fails, a random variable $(\alpha)$ gives the location of the failure as shown in Figure 1 . Thus when a technician has to repair a link failure, the traveling distance is the sum of the distance from his initial location to the node closer to the failure plus the distance from this node to the failure.

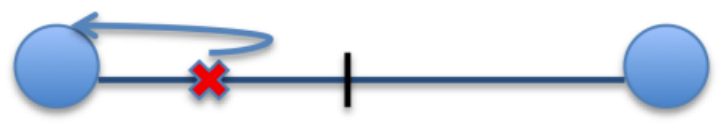

$$
\begin{aligned}
& \text { Assigned node }= \begin{cases}A & \text { if } \alpha<0,5 \\
B & \text { if } \alpha>0,5\end{cases} \\
& \text { Added distance }= \begin{cases}\alpha * l & \text { if } \propto<0,5 \\
(1-\propto) * l & \text { if } \propto>0,5\end{cases}
\end{aligned}
$$

Node A

$\propto=0,25$

Node $B$

Figure 1 Link failure location

The technician location problem has been identified as an Integer Linear Programming (ILP) problem; ILP is a technique for optimization of a linear objective function, subject to linear equality and linear inequality constraints. According with previous information and knowing data input, the objective function has been defined such as follows:

$\min \left\{\sum_{i}^{N} w * T_{i}+\sum_{i}^{N} \sum_{j}^{N}\left[P_{n} *\left(\frac{d_{i j}}{s}+R_{n}\right) * F_{j}^{n}+P_{l} *\left(\frac{d_{i j}}{s}+\frac{d_{\text {ext }}}{s}+R_{l}\right) * F_{j}^{l}\right] * x_{i j}\right\}$

$w=$ wage, salary per technician

$T_{i}=\left\{\begin{array}{l}1 \\ 3\end{array}\right.$ if technicians in node $i$ has to repair just node failures

$T_{i}=\left\{\begin{array}{l}1 \\ 0\end{array} \quad\right.$ if technicians in node $i$ have to repair link failures

$P_{n}=$ node penalty, cost per hour without service

$P_{l}=$ link penalty, cost per hour without service

$d_{i j}=$ distance between technician in node $i$ to fail in node $j$

$s=$ average speed

$R_{n}=$ reparation time of one node

$R_{l}=$ reparation time of one link

$d_{\text {ext }}=$ distance from link failure to associated node

$F_{j}^{n}=$ number of failures in node $j$

$F_{j}^{l}=$ number of failures in link associated to node $j$

$x_{i j}=\left\{\begin{array}{rr}1 & \text { if technician in node i repair fail in node } j \\ 0 & \text { otherwise }\end{array}\right.$

The first term $w * T_{i}$, tries to minimize the costs of technicians at node $i$, subject to salary per technician $(w)$ and the number of technicians $T_{i}$ required to repair a failure. It has been assumed that one technician is required for node reparation, whereas 3 technicians are required for link reparation.

The second term is more complex. Let us give some explanation:

- This term includes inputs referred to node failures (marked with " $n$ ") and inputs referred to link failures (marked with "l").

- It includes some shared inputs such as distance between nodes and the average traveling speed. These parameters are static and have the same value regardless of the type of failure (node or link).

- At the end of this term, the variable $x_{i j}$ informs about whether technician at node $i$ should repair the failure associated to node $j$.

- $\quad P_{n}$ and $P_{l}$ give the penalty cost per time for a node or link failure. This penalty is given in cost per hour and should be multiplied by the service interruption time associated to each failure. This time is the sum of the traveling time from the closest technician location to the failure and the reparation time, which depends on the type of failure: $R_{n}$ for nodes and $R_{l}$ for links). The traveling time can be obtained dividing the traveling distance by the speed (i.e. $d_{i j} / s$ when the failure is in node $\mathrm{j}$ but $d_{i j} / s+d_{e x} / s$ when the failure is in a link associated to node $\mathrm{j}$ and is located $d_{e x t} \mathrm{~km}$ farther from it).

- $\quad F_{j}^{n} * x_{i j}$ and $F_{j}^{l} * x_{i j}$ : the number of failures in node $j$ or in link associated to node $j$, times the binary variable, which is 1 if technician in node $i$ has to repair failure in node $j$. 
In order to establish limits and achieve a real optimized result, this problem requires a set of constraints, which are shown below:

1. All failures have to be repaired:

$\sum_{i}^{N} \sum_{j}^{N} x_{i j} *\left(F_{j}^{n}+F_{j}^{l}\right)=F_{\text {tot }} ; F_{\text {tot }}=$ Total number of failures

2. Each node is repaired just for one technician:

$\sum_{i}^{N} x_{i j}=1$

3. Enough technicians to repair all failures (node and link):

Auxiliary expressions $\left\{\begin{array}{l}a=\sum_{j=0}^{N} x_{i j} * F_{j}^{l} \\ b=\sum_{j=0}^{N} x_{i j} * F_{j}^{n}\end{array}\right.$

According with previous auxiliary expressions, the $T_{i}$ constraint can be defined as:

$T_{i}=3 * a_{b i n}+1 * a u x$

where aux is defined below:

$\operatorname{aux} \leq \frac{b_{\text {bin }}-a_{\text {bin }}+1}{2}$

$a u x \geq b_{\text {bin }}-a_{\text {bin }}$

In order to address the optimization problem is very important to use the suitable tools. The tools used for this implementation are: Java and Gurobi, which work together to solve the optimization problem.

\section{PARTICULAR STUDY CASE}

The implementation of the problem can be applied to any network, any topology with any characteristics (failure rates, traveling speed, penalties, etc.)

As an example, the proposed formulation has been applied to the Germany50 network [2]. The Germany50 network has 50 nodes and 88 links.

\subsection{Input data}

The input data can be divided in three kinds of variables: time, cost and failure variables.

As time variable, the travelling time and time to repair each failure should be defined. The travelling time is the ratio of the distance to be travelled by the technician(s) and the speed. The distance is given by the coordination of the nodes. The average speed has been assumed to be $80 \mathrm{~km} / \mathrm{h} \mathrm{[3]}$. On the other hand, the reparation time of a failure depends on the type of failure and has been assumed to be one hour for a node failure and seven hours for a link failure [3].

As cost variables, the technician salary and the penalty for connection interruption time should be given. The average technician salary has been assumed to be $140 €$ /hour and the penalty varying from 10 to $1000 € /$ hour [3]. The operational time of the network has been assumed to be 20 years, so that for this period, the salary cost per technician is proximately $5.367 .000 €$ and the penalty varies between from 1000 to $10000000 €$.

The failure variables depend on the type of failure (node or link). Based on some references [4][5] of previous studies, the FIT bounds of a node can vary from 10.000 for an opaque optical node and 560 for new optical cross connects (OXCs). On the other hand, the bounds for FIT of the link have been established in $310 \mathrm{FIT} / \mathrm{km} \mathrm{[5]} \mathrm{as} \mathrm{lower} \mathrm{limit} \mathrm{and} 570 \mathrm{FIT} / \mathrm{km}$ [3] as higher limit.

\subsection{Study results}

The result of this study is given as the number of technicians and their locations, as well as the total cost of failure reparation process for different values of their penalty (shown in Figure 2 and 3 respectively). For each study, two extreme failure rate cases have been considered: High failure rate (10000 FIT for nodes and 570FIT/km for link) and low failure rate (560 FIT for node and $310 \mathrm{FIT} / \mathrm{km}$ for link). It can be observed that for low penalties, the number of technicians is low, since having low number of technicians imply having low salary costs but longer service interruption times. But as penalty cost increase, the solution gives more technicians so that the time to repair failures decreases (as well as the related penalties). At some penalty value (lower for high failure rates) the number of technicians is maximum 
(150, which refers to the case of having 3 technicians at every network node). Regarding the total cost, it can be observed that the failure reparation cost is extremely high when the penalty cost is above $1000000 € / \mathrm{h}$ since the reparation time cannot be further reduced (already maximum number of technicians).

Furthermore, a sensitivity analysis has been performed when varying the failure rate of node and links as shown in Figures 4 and 5. It can be observed how the total cost as well as the number of technicians increase with the failure rate.
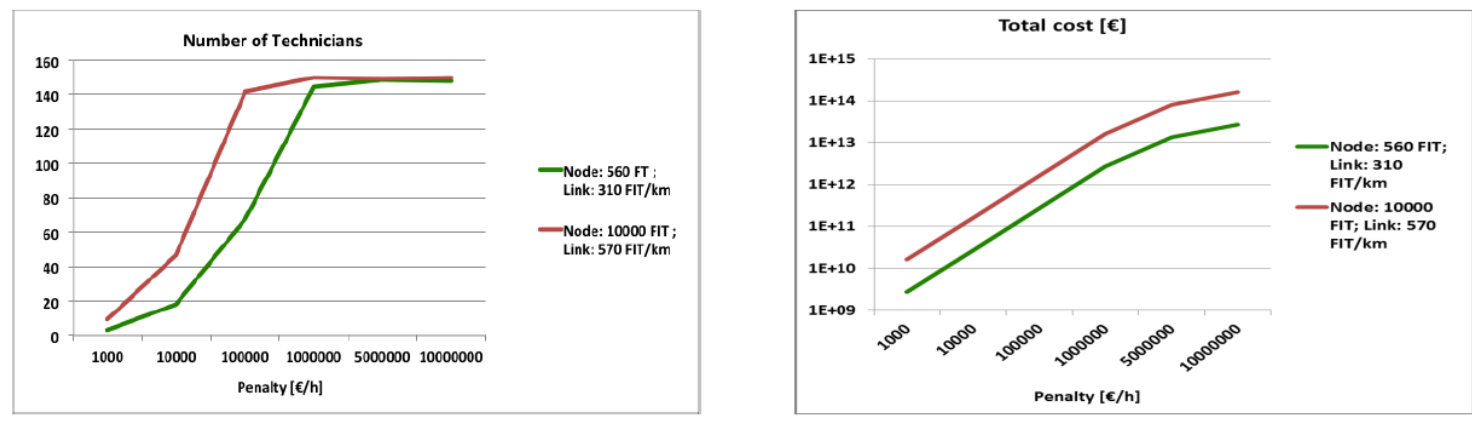

Figure 2. Number of technicians for different penalties

Figure 3. Total failure reparation cost for different penalties

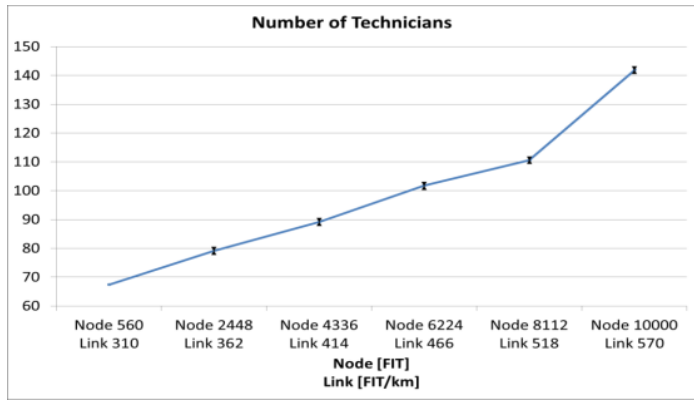

Figure 4. Number of technicians varying failure rate

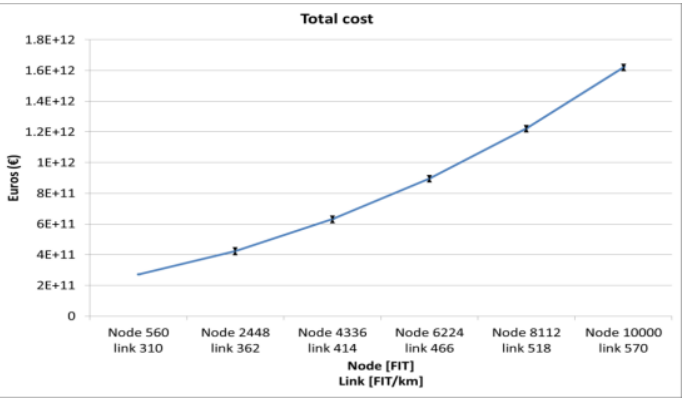

Figure 5 Total cost varying failure. rate

\section{CONCLUSIONS}

In this article the technician location optimization problem has been presented targeting to minimize time and cost of repairing all failures. The obtained results give us the opportunity of understanding how the cost and number of technicians vary depending on the input parameters. Looking at the particular case study on Germany50 network, it can be seen that, higher penalty implies higher fault reparation cost. When the penalty is getting comparable to the salary for the whole network operational time, is better to place more technicians in order to minimize the total cost. On the other hand, it can also be observed that higher FIT implies more failures therefore more penalties and more technicians, which increase the total cost of repairing the network.

\section{REFERENCES}

[1] C. Mas Machuca, O. Moe, M. Jäger „Modelling of OpEx in network and service life-cycles” ECOC 2007 OpEx Workshop, Berlin, Germany, September 2007

[2] SNDLib problem instances: sndlib.zib.de/

[3] J. Chen, L. Wosinska, C. Mas Machuca, M. Jaeger "Cost vs. Reliability Performance Stud of Fiber Access Network Architectures” IEEE Communications Magazine, February 2010.

[4] T. Bedford "Safety and reliability. Improvement of risk -based methodologies through the combination of technical, project, financial and environmental approaches to risk" Proc. ESREL 2003.

[5] D. A. A. Mello, D. A. Schupke, M. Scheffel and H. Waldman. Availability Maps for Connections in WDM Optical Networks. Design of Reliable Communication Networks (DRCN) October 2005. 